\title{
Little clinical benefit of early systemic aciclovir for treatment of primary herpetic stomatitis
}

\author{
Is systemic aciclovir effective in the treatment of primary herpetic stomatitis?
}

\author{
Nasser M, Fedorowicz Z, Khoshnevisan MH, \\ Shahiri Tabarestani M. \\ Acyclovir for treating primary herpetic gingivostomatitis. Cochrane \\ Database Syst Rev 2008; issue 4
}

Data sources The Cochrane Oral Health Group's Trials Register; Cochrane Central Register of Controlled Trials, Medline and Embase were used to search for studies. There were no language restrictions.

Study selection Randomised controlled trials (RCT) were eligible if they compared aciclovir to placebo in children and young adults ( $<25$ years of age) who had been diagnosed with primary herpetic gingivostomatitis with or without herpes labialis.

Data extraction and synthesis Two review authors independently and in duplicate screened and extracted information from, and assessed the risk of bias, in the included clinical trials. The Cochrane Collaboration statistical guidelines were followed for data synthesis.

Results Only two clinical trials, one with 72 participants and the other with 20, were included in this review. The second study failed to report details of its methods and was inconsistent in its reporting of the outcomes measured. The first trial, which had a moderate risk of bias, showed that aciclovir was better than placebo at reducing, in children of $<6$ years, the number with oral lesions [risk ratio (RR), 0.10; $95 \%$ confidence interval $(\mathrm{Cl}), 0.02-0.38)$ ], with new extra-oral lesions (RR; 0.04; $95 \% \mathrm{Cl}, 0.00-0.65)$, and difficulty in eating (RR, $0.14 ; 95 \% \mathrm{Cl}, 0.03-$ $0.58)$ and drinking (RR, $0.11 ; 95 \% \mathrm{Cl}, 0.01-0.83)$ after 8 days of treatment. Following onset of treatment, three patients from the placebo group were admitted to hospital for rehydration ( $P 0.11)$. Four children (two from the aciclovir group and two taking placebo) suffered mild gastrointestinal symptoms that resolved spontaneously after $24-48 \mathrm{~h}$ without a change in the study regimen.

Conclusions Two relevant trials were found for this systematic review. Only one was able to provide weak evidence that aciclovir is an effective treatment in children under 6 years of age with primary herpetic gingivostomatitis, in reducing the number of oral lesions, preventing the development of new extra-oral lesions, decreasing the number of individuals experiencing difficulties eating and drinking and reducing hospital admission.

\section{Commentary}

Systemic aciclovir for the treatment of primary herpetic gingivostomatitis in immunocompetent children and young adults is rarely warranted as the disease is generally self-limiting and rarely gives rise to systemic complications. In contrast, antiviral therapy for herpes simplex virus (HSV) infection in immunocompromised children and adults is essential to reduce the risk of both local and systemic complications. The results of the present systematic review indicate that there is some, but only a little, significant clinical benefit of early systemic aciclovir for this disease in children aged under 6 years but, notably, there are few relevant studies. The report reveals no striking new trends that have not been described in other less stringent investigations (eg, retrospective analyses, open studies) and thus it simply confirms what is known from current literature, and practised by most informed clinicians.

The authors suggest that there is a need for more appropriately planned trials of the efficacy of aciclovir in age groups other that young children. This is certainly worthy of consideration, particularly as there has been a striking rise in the prevalence of primary orofacial herpetic infection in young adults in the developed world. A more detailed review of the advances in antiviral strategies might have indicated that aciclovir may not be the most appropriate agent to consider for such studies. The focus of any study should also surely be the treatment of immunocompromised rather than immunocompetent groups: such investigations are likely to be costly and should first focus upon disease of greatest clinical need.

\section{SR Porter}

University College London Eastman Dental Institute, London, UK

Evidence-Based Dentistry (2008) 9, 117. doi:10.1038/sj.ebd.6400617 